\title{
Investigation of the Need for Computed Tomography Pulmonary Angiography in the Decision to Discontinue Treatment for Pulmonary Thromboembolism
}

\section{Eda Çelik ( $\sim$ edakguney@hotmail.com )}

Ağrı İbrahim Çeçen Üniversitesi: Agri Ibrahim Cecen Universitesi https://orcid.org/0000-0002-95902204

\section{Ömer Araz}

Ataturk University: Ataturk Universitesi

\section{Buğra Kerget}

Ataturk University: Ataturk Universitesi

\section{Elif Yılmazel Uçar}

Ataturk University: Ataturk Universitesi

\section{Metin Akgün}

Ataturk University: Ataturk Universitesi

\section{Leyla Sağlam}

Ataturk University: Ataturk Universitesi

\section{Research Article}

Keywords: Computed tomography, pulmonary thromboembolism, PAOI

Posted Date: December 6th, 2021

DOI: https://doi.org/10.21203/rs.3.rs-1127219/v1

License: (a) (i) This work is licensed under a Creative Commons Attribution 4.0 International License. Read Full License 


\section{Abstract}

Purpose: Acute pulmonary thromboembolism (PTE) is an important cause of morbidity and mortality that can reduce quality of life due to long-term complications during and after treatment discontinuation. The aim of this study was to evaluate patients for these complications before discontinuing treatment and determine the necessity of computed tomography pulmonary angiography (CTPA) imaging.

Methods: This retrospective study included 116 patients over the age of 18 who received anticoagulant treatment for at least 3 months and presented for treatment discontinuation to the Atatürk University Research Hospital Chest Diseases Outpatient Clinic between January 2015 and September 2019.

Results: CTPA performed at treatment discontinuation showed complete thrombus resolution with treatment in 73 patients (62.9\%). High pulmonary artery obstruction index (PAOI) at diagnosis was statistically associated with findings of residual or chronic thrombus on CTPA at treatment discontinuation $(p=0.001)$. In the differentiation of patients with residual/chronic thrombus and those with thrombus resolution, D-dimer at a cut-off value of $474 \mu \mathrm{g} / \mathrm{L}$ had $60 \%$ sensitivity and $70 \%$ specificity. At a cut-off value of $35.5 \mathrm{mmHg}$, mean pulmonary artery pressure on echocardiography had sensitivity and specificity of $72 \%$ and $77 \%$, respectively. At a cut-off of 23.75 , PAOI had sensitivity and specificity of $93 \%$ and $69 \%$, respectively.

Discussion: In addition to physical examination findings, D-dimer and echocardiography were guiding parameters in the evaluation of treatment discontinuation and thrombus resolution in patients presenting to the outpatient clinic for discontinuation of treatment for acute PTE. PAOI at diagnosis may be another important guiding parameter in addition to these examinations.

\section{Introduction}

Pulmonary thromboembolism (PTE) generally occurs as a complication of deep vein thrombosis (DVT) when the pulmonary artery and/or its branches are occluded by clots that break off from thrombi in the deep veins of the leg. As PTE and DVT often occur together, the term venous thromboembolism (VTE) can also be used to describe both events [1].

After a first episode of VTE, the patient is examined for possible risk factors. In cases of VTE associated with reversible or transient risk factors such as immobilization, medical illness, estrogen therapy, pregnancy, surgery, or trauma, prophylaxis therapy for three months or longer (six months) is recommended [2]. For patients with two or more recurrent episodes of idiopathic or thrombophilic VTE, at least 12 months or continuous prophylaxis is recommended. Cancer patients have a high risk of VTE and its recurrence. Even at the first episode, these patients should receive long-term anticoagulant therapy until resolution is achieved $[3,4]$.

Monitoring the residual thrombus by venous Doppler ultrasonography during secondary prophylaxis aids in determining treatment duration. High D-dimer levels or the presence of residual venous thrombosis 
after oral anticoagulant therapy are important predictors of recurrence [5]. When discontinuing treatment in PTE, the evaluation of symptoms, D-dimer level, echocardiography (ECHO), and bilateral lower extremity color Doppler ultrasound (US) findings can help identify patients who should be considered at risk of recurrence. Guidelines recommend computed tomography pulmonary angiography (CTPA) when discontinuing treatment in symptomatic patients [6]. However, some patients with no clinical symptoms have positive imaging results. This is important for the evaluation of chronic thromboembolism. As there is no clear statement regarding treatment discontinuation in our national guidelines, CTPA is generally performed routinely in all patients at the end of treatment [7]. However, this may cause unnecessary exposure to radiation and contrast agent in some patients.

Therefore, this study aimed to compare CTPA findings obtained before discontinuing treatment with symptoms, D-dimer, ECHO, and pulmonary artery obstruction index (PAOI) findings to evaluate in which cases CTPA should be performed.

\section{Materials And Methods}

\section{Study design}

We retrospectively reviewed the data of patients diagnosed with PTE who had received the required duration of anticoagulant therapy according to their risk factors and presented for treatment discontinuation to the Atatürk University Chest Diseases Outpatient Clinic between January 2015 and September 2019. Patient data were collected from the hospital information system and outpatient clinic records after obtaining ethics committee approval (dated 26.09.2019, number B.30.2.ATA.0.01.00/548).

\section{Study population}

The study included 116 patients registered in the Atatürk University Chest Diseases Outpatient Clinic who were over 18 years of age and had received anticoagulant therapy for at least 3 months after being diagnosed as having acute PTE. Patients who did not have CTPA, D-dimer, bilateral lower extremity venous Doppler US, or ECHO data from the time of diagnosis or treatment discontinuation, had poor treatment compliance, were pregnant at the time of treatment discontinuation, had renal failure or any obstructive or restrictive pulmonary pathology, or were followed up with V/Q scintigraphy were excluded from the study.

\section{Follow-up parameters}

The patients' age and gender were recorded. The data were reviewed to determine whether the patient had any predisposing factor (idiopathic or non-idiopathic). If non-idiopathic, information regarding any surgical procedure within one month before diagnosis, history of trauma, and whether the patient had any neurological disease causing immobilization, malignancy, pregnancy, or used oral contraceptives at the time of diagnosis were recorded. Additionally, at the time of diagnosis radiologists examined the main femoral vein, deep femoral vein, superficial femoral vein, and popliteal vein in both lower extremities using color Doppler US while the patients were in supine position. Vein enlargement, noncompressibility, 
absence of flow on color Doppler, and direct observation of a thrombus were regarded as signs of DVT. The results of these examinations were obtained from the hospital information system and we noted whether the patient had DVT and the thrombus location (main femoral vein, superficial femoral vein, deep femoral vein, popliteal vein) if present. We also reviewed the hospital information system to obtain the results of the same examinations performed for each patient before discontinuing treatment.

With patients in supine position, intravenous contrast agent (iopromide) was administered using an automatic CT injector and CTPA imaging was performed with multidetector computed tomography during the pulmonary artery phase. We reviewed the results of radiologists' CTPA examinations performed at the time of diagnosis and recorded the thrombus localization on CTPA as main pulmonary artery, lobar pulmonary artery branch, or segmental pulmonary artery branch. Similarly, we reviewed the reports from CTPA examinations obtained using the same method before treatment discontinuation and recorded whether the patient had no thrombus, thrombus in the main pulmonary artery, thrombus in the lobar pulmonary artery, thrombus in the segmental pulmonary artery, or chronic embolism. CTPA findings other than intraluminal filling defect such as the thrombus being parallel to and forming an obtuse angle with the vessel wall; post-stenotic dilatation or aneurysm; sequela changes such as web or bands; mosaic perfusion; recanalization or calcification in the thrombus were regarded as indicative of chronic thrombus. Otherwise, the presence of intraluminal filling defect alone was interpreted as suggestive of residual thrombus.

We recorded the patients' mean pulmonary arterial pressure (MPAP) values calculated by cardiologists based on tricuspid regurgitation in ECHO performed with the patient in left lateral decubitus position before treatment discontinuation. An mPAP value of $40 \mathrm{mmHg}$ or higher was considered pathological. The patients' serum D-dimer levels before treatment discontinuation were recorded. D-dimer level was measured by immunofluorescence assay and interpreted as pathological at $500 \mu \mathrm{g} / \mathrm{L}$ or higher. We also recorded the patients' duration of treatment, what treatment they received during follow-up (low molecular weight heparin [LMWH], warfarin, direct oral anticoagulant [DOAC]), and whether they received thrombolytic therapy at the time of diagnosis. Thrombolytic therapy consisted of alteplase administered at a dose of $100 \mathrm{mg}$ or $50 \mathrm{mg}$. Finally, we determined from outpatient records whether the patients had complaints of dyspnea at the time of treatment discontinuation.

\section{Pulmonary Artery Obstruction Index (Paoi)}

PAOI was determined from CTPA images by first dividing the pulmonary arteries into 10 segmental branches for each lung ( 3 in the upper lobe, 2 in the middle lobe and lingula, 5 in the lower lobe). Thrombi in proximal pulmonary arteries (main, lobar) were assigned a value $n$ equal to the sum of all segmental branches distal of the thrombus (min. 1-max. 20). In the absence of a thrombus in the proximal pulmonary arteries, isolated thrombi in segmental branches were assigned an $n$ value of 1 point. Degree of obstruction $(d)$ was scored as 1 for partial obstruction (filling defect with flow of contrast agent past 
the thrombus) and 2 for total obstruction (absence of any contrast agent distal to the thrombus). PAOI was calculated as a percentage of the maximum total possible score of 40 using the following equation:

$\mathrm{PAIO}=\Sigma(n \times d) / 40 \times 100$.

\section{Statistical analysis}

The data were analyzed using IBM SPSS version 20.0 statistical software. Descriptive statistics were calculated to summarize the data. The data were tested for normal distribution using the Shapiro-Wilk test when the sample size was less than 5 and the Kolmogorov-Smirnov test when the sample size was 5 or more. Comparisons between two independent groups were made with Mann-Whitney, Kruskal-Wallis, and Wilcoxon tests if the data were not normally distributed. The Kruskal-Wallis test with Bonferroni correction was used to identify variables that were significantly different. In 2x2 comparisons between categorical variables, Pearson's chi-square test was used if the expected value was greater than 5, Yates' chi-square test was used if the expected value was between 3 and 5, and Fisher exact test was used if the expected value was less than 3 . Correlations between pairs of quantitative variables were analyzed using Pearson correlation analysis if the data were normally distributed and Spearman correlation analysis if not. Receiver operating characteristic (ROC) curve analysis was used to determine the diagnostic value of continuous variables. The Youden index was used to determine optimal cut-off values. The level of statistical significance was set at $5 \%$.

\section{Results}

The mean age of the 116 patients included in the study was $62.1 \pm 16.1$ years. Sixty-four $(55.2 \%)$ of the patients were women. The mean ages of the women and men were $62.8 \pm 16.5$ and $61.3 \pm 15.7$ years, respectively $(p=0.62)$.

The patients' demographic data, CTPA findings at time of diagnosis and treatment discontinuation, treatment duration, and treatment modalities are shown in Table 1. On CTPA performed at diagnosis, thrombus was most commonly located in the main pulmonary artery $(n=56,48.3 \%)$. On CTPA examination at treatment discontinuation, 73 patients $(62.9 \%)$ had no thrombus. Treatment duration was 6 months or less in 86 patients (74.1\%). Seventy-one patients (61.2\%) of the patients received LMWH, 21 (18.1\%) received warfarin, and $24(20.7 \%)$ received DOACs. 
Table 1

Patient demographic characteristics, radiological findings, and treatments received during follow-up

$\mathrm{n}(\%)$

Sex

Male

$52(44.8)$

Female

$64(55.2)$

Age (years)

$62.1 \pm 16.1$

Thrombus location on CTPA at diagnosis

Main Pulmonary Artery

$56(48.3)$

Lobular Pulmonary Artery

$23(19.8)$

Segmental Pulmonary Artery

$37(31.9)$

Thrombus features on CTPA at treatment discontinuation

No thrombus detected

$73(62.9)$

Thrombus in the main pulmonary artery

$6(5.2)$

Thrombus in the lobar pulmonary artery

$3(2.6)$

Thrombus in segmental pulmonary artery

19 (19.4)

Chronic thrombus

$15(12.9)$

Treatment duration

$\leq 6$ months

$86(74.1)$

$>6$ months

$30(25.9)$

Thrombolytic therapy

Not given

79 (68.1)

$100 \mathrm{mg}$

21 (18.1)

$50 \mathrm{mg}$

$24(20.7)$

Treatment received

LMWH

71 (61.2)

Warfarin

21 (18.1)

DOAC

21 (20.7)

CTPA: Computed tomography pulmonary angiography, LMWH: Low molecular weight heparin, DOAC: Direct oral anticoagulant 
Analysis of the presence and location of thrombi detected on CTPA at treatment discontinuation according to thrombus location on CTPA at diagnosis, PAOI values, D-dimer level at treatment discontinuation, and abnormal PAP on ECHO, and presence of dyspnea are shown in Table 2. CTPA performed at treatment discontinuation demonstrated complete resolution with treatment in 73 (62.9\%) of the 116 PTE patients. We observed no significant differences in our statistical analysis of patients with residual and chronic thrombus according to thrombus location on CTPA at diagnosis $(p=0.258)$. A greater proportion of patients with thrombus resolution were below the thresholds of $500 \mu \mathrm{g} / \mathrm{L}$ for D-dimer level at treatment discontinuation $(p=0.001)$ and $40 \mathrm{mmHg}$ for mPAP on ECHO $(p=0.001)$. In addition, chronic and residual thrombus were less common in patients without dyspnea $(p=0.005)$. Patients with chronic thrombus had higher PAOI at diagnosis $(p=0.001)$. However, there was no significant difference in PAOI at treatment discontinuation between patients with residual and chronic thrombus $(p=0.45)$.

Table 2

Comparison of CTPA at treatment discontinuation with CTPA at diagnosis, laboratory results, and PAOI values

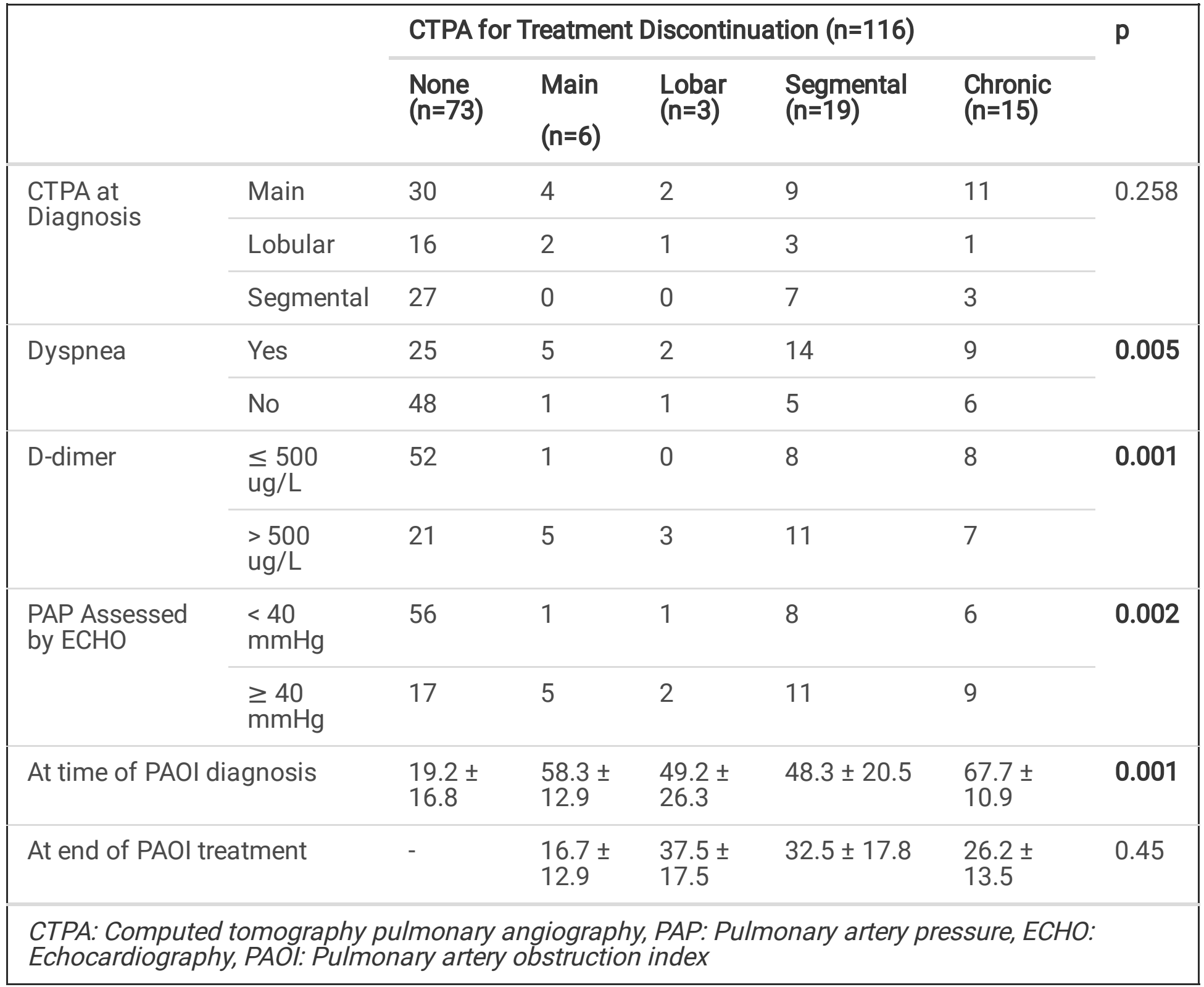


Among patients with thrombus resolution on CTPA, mPAP assessed by ECHO was below $40 \mathrm{mmHg}$ in 56 patients and above $40 \mathrm{mmHg}$ in 17 patients. Of those with residual or chronic thrombus on CTPA, mPAP was below $40 \mathrm{mmHg}$ in 16 patients and above $40 \mathrm{mmHg}$ in 27 patients.

ROC curve analysis of D-dimer, mPAP on ECHO, and PAOI at diagnosis in patients with residual/chronic thrombus and patients with thrombus resolution are shown in Figure 1. D-dimer at a cut-off value of 474 $\mu \mathrm{g} / \mathrm{L}$ had sensitivity and specificity of $60 \%$ and $70 \%$, respectively. Mean pulmonary artery pressure on $\mathrm{ECHO}$ at a cut-off value of $35.5 \mathrm{mmHg}$ had sensitivity and specificity of $72 \%$ and $77 \%$, respectively. At a cut-off value of 23.75 , PAOI had sensitivity and specificity of $93 \%$ and $69 \%$, respectively.

\section{Discussion}

Of the 116 patients in our study, CTPA at treatment discontinuation demonstrated thrombus resolution in 73 patients. The risk of residual and chronic thrombus was higher in patients with a D-dimer level higher than $500 \mu \mathrm{g} / \mathrm{L}$ and mPAP above $40 \mathrm{mmHg}$ at treatment discontinuation. Our analysis of the relationship between thrombus on CTPA and dyspnea revealed that chronic and residual thrombus were less common in patients without dyspnea. In addition, we determined that high PAOI at time of diagnosis was associated with higher risk of chronic and residual thrombus. In addition, ROC curve analysis of D-dimer, mPAP, and PAOI at diagnosis showed that PAOI had higher sensitivity in detecting chronic and residual thrombus than the other two parameters.

PTE is one of the major emergencies for which morbidity and mortality rates are declining due to advances in early diagnosis and treatment [8]. In treatment, the duration of secondary prophylaxis varies based on the risk factors present [9-11]. It was reported that $81 \%$ of 69 PTE patients followed up with CT angiography had complete resolution after 4 weeks [12]. For this reason, follow-up with CTPA is not routinely done in patients being treated for PTE. However, this degree of complete resolution is seen in $50-80 \%$ of cases. Patients should be evaluated for long-term complications of PTE when discontinuing treatment [13].

As in acute PTE, dyspnea is one of the main symptoms of chronic thromboembolic pulmonary hypertension (CTEPH). A multicenter, prospective observational study aiming to develop a dyspnea-based screening algorithm to predict the incidence of CTEPH was conducted in 1699 acute PTE patients receiving treatment between March 2009 and November 2016 and published in 2018. In the study, patients were assessed by phone using a dyspnea questionnaire at 6,12 , and 24 months. Patients whose dyspnea complaints were class II or higher according to the New York Heart Association (NYHA) Functional Classification underwent ECHO examination. Radiological imaging (CTPA or V/Q scintigraphy) was performed in patients with suspected CTEPH based on these two steps. The negative predictive value of the first two steps was reported to be $99 \%$. In the study, a significant statistical relationship was observed between dyspnea complaints and on follow-up imaging findings [14]. 
Consistent with the results of that study, we observed that dyspnea was more common in patients with chronic and residual thrombus.

D-dimer is the smallest fibrin degradation product formed by the breakdown of cross-linked fibrin by plasmin. Its presence confirms both thrombin and plasmin formation. Serum D-dimer levels increase due to the simultaneous coagulation and fibrinolysis processes that occur in acute thromboses and are expected to regress with resolution of the thrombus in acute PTE $[15,16]$. In a 2006 study of 608 acute PTE patients followed up with oral anticoagulant therapy for 3 months, treatment was discontinued in patients with normal D-dimer levels, while patients with high D-dimer levels were randomly assigned to either discontinue or continue treatment. Examinations performed 1 month later showed that patients with high D-dimer levels who discontinued treatment had a significantly higher recurrence rate than those who continued treatment. This study supported the significance of monitoring D-dimer level in determining the duration of acute PTE treatment [17].

$\mathrm{ECHO}$ is another examination commonly used to evaluate the likelihood of recurrent and chronic thrombus in acute PTE or PTE follow-up. The effects of pulmonary hypertension on the heart are evaluated by ECHO and mPAP is estimated by continuous wave Doppler measurements. The determination of mPAP is based on the maximal tricuspid regurgitation velocity and right atrial pressure. Some preoperative studies conducted in CTEPH patients demonstrated a correlation between the mPAP assessed by ECHO and mPAP assessed by catheterization [18]. Therefore, ECHO is a useful, noninvasive method for the follow-up of long-term PTE complications. The center in which our study was conducted is one of the few high-altitude settlements in our country and the world, and elevated mPAP is frequently observed in our region due to chronic hypoxia. Using previous studies as a reference, an mPAP threshold of $40 \mathrm{mmHg}$ was used in this study [19].

According to the data obtained in our study, we observed no statistically significant relationship between CTPA performed at diagnosis and the later development of chronic thrombus and residual thrombus. This suggests that chronic and residual thrombus evaluation should be done during follow-up on an individual basis and that even if initial thrombus load is high, the development of chronic and residual thrombus may be related to the patient, independent of treatment. In our evaluation of D-dimer and mPAP on ECHO, which are parameters commonly used in the diagnosis and treatment of PTE, we found that the probability of residual and chronic thrombus was lower if D-dimer level was below $500 \mu \mathrm{g} / \mathrm{L}$ and mPAP was below $40 \mathrm{mmHg}$. These findings may be attributed to a lower thrombus load resulting in both reduced D-dimer level, which is an indicator of fibrin degradation, and decreased right heart load. Our ROC analysis of PAOI at diagnosis of PTE and D-dimer level and mPAP at treatment discontinuation showed that PAOI was a better predictor of chronic and residual thrombus development than the other two parameters. An increase in the mPAP measured on ECHO may be triggered by many comorbidities and there may be differences between measurements. In our study, we tried to exclude conditions that may cause this. Similarly, although D-dimer level can serve as a guiding parameter in cases of suspected PTE, its level is affected by most comorbidities independent of thrombus load. As opposed to these, PAOI 
seems to have value as a better indicator of thrombus load. Therefore, we believe that priority should be given to patients with high PAOI at time of diagnosis when planning CTPA during follow-up.

This study has certain limitations. Most importantly, because this was a retrospective study, patients classified as having idiopathic PTE could not be evaluated for permanent risk factors and thrombophilia, and we were unable to obtain sufficient data regarding these parameters. Furthermore, because NYHA functional class was not used during dyspnea assessment, a detailed inquiry was not possible.

Based on the results of this study, we believe that evaluation with CTPA at treatment discontinuation is necessary in patients with elevated D-dimer, mPAP on ECHO above $40 \mathrm{mmHg}$, and especially high PAOI at diagnosis.

Ackknowledgement None to declare

Statement of Ethics Local ethics committee approved the study.

\section{Disclosure}

Statement None

\section{Declarations}

Ackknowledgement None to declare

Statement of Ethics Local ethics committee approved the study.

Disclosure Statement None

Funding Sources None.

\section{References}

1. Wood KE (2002) Major pulmonary embolism: review of a pathophysiologic approach to the golden hour of hemodynamically significant pulmonary embolism. Chest 121(3):877-905

2. Bělohlávek J, Dytrych V, Linhart A (2013) Pulmonary embolism, part I: Epidemiology, risk factors and risk stratification, pathophysiology, clinical presentation, diagnosis and nonthrombotic pulmonary embolism. Experimental \& Clinical Cardiology 18(2):129

3. Svendsen E, Karwinski B (1989) Prevalence of pulmonary embolism at necropsy in patients with cancer. J Clin Pathol 42(8):805-809

4. Tapson VF (2008) Acute pulmonary embolism. N Engl J Med 358(10):1037-1052

5. Stein PD, Hull RD, Patel KC, Olson RE, Ghali WA, Brant R, Biel RK, Bharadia V, Kalra NK (2004) D-dimer for the exclusion of acute venous thrombosis and pulmonary embolism: a systematic review. Ann Intern Med 140(8):589-602 
6. Ucar EY (2019) Update on thrombolytic therapy in acute pulmonary thromboembolism. The Eurasian journal of medicine 51(2):186

7. Konstantinides SV, Meyer G, Becattini C, Bueno H, Geersing G-J, Harjola V-P, Huisman MV, Humbert M, Jennings CS, Jiménez D (2020) 2019 ESC Guidelines for the diagnosis and management of acute pulmonary embolism developed in collaboration with the European Respiratory Society (ERS) The Task Force for the diagnosis and management of acute pulmonary embolism of the European Society of Cardiology (ESC). Eur Heart J 41(4):543-603

8. Yilmazel Ucar E, Araz O, Kerget B, Yilmaz N, Akgun M, Saglam L (2018) Comparison of long-term outcomes of 50 and $100 \mathrm{mg}$ rt-PA in the management of acute pulmonary thromboembolism. Clin Respir J 12(4):1628-1634

9. Kerget B, Afşin DE, Aksakal A, Aşkin S, Araz Ö (2020) Could HIF-1 $\alpha$ be a novel biomarker for the clinical course and treatment of pulmonary embolism? Turk J Med Sci 50(4):963-968

10. Ucar EY, Akgun M, Araz O, Tas H, Kerget B, Meral M, Kaynar H, Saglam L (2015) Comparison of LMWH versus UFH for hemorrhage and hospital mortality in the treatment of acute massive pulmonary thromboembolism after thrombolytic treatment. Lung 193(1):121-127

11. Kerget B, Afşin DE, Aksakal A, Kerget F, Aşkın S, Ucar EY, Sağlam L (2021) Could VEGF-D level have a role in clinical risk scoring, estimation of thrombus burden, and treatment in acute pulmonary thromboembolism?

12. Stein PD, Yaekoub AY, Matta F, Janjua M, Patel RM, Goodman LR, Gross ML, Denier JE (2010) Resolution of pulmonary embolism on CT pulmonary angiography. Am J Roentgenol 194(5):12631268

13. Korkmaz A, Ozlu T, Ozsu S, Kazaz Z, Bulbul Y (2012) Long-term outcomes in acute pulmonary thromboembolism: the incidence of chronic thromboembolic pulmonary hypertension and associated risk factors. Clin Appl Thromb Hemost 18(3):281-288

14. Coquoz N, Weilenmann D, Stolz D, Popov V, Azzola A, Fellrath J-M, Stricker H, Pagnamenta A, Ott S, Ulrich S (2018) Multicentre observational screening survey for the detection of CTEPH following pulmonary embolism. European respiratory journal51 (4)

15. Kearon C, Ginsberg JS, Douketis J, Turpie AG, Bates SM, Lee AY, Crowther MA, Weitz JI, Brill-Edwards P, Wells $P$ (2006) An evaluation of D-dimer in the diagnosis of pulmonary embolism: a randomized trial. Ann Intern Med 144(11):812-821

16. Perrier A, Desmarais S, Goehring C, de MOERLOOSE P, Morabia A, Unger P-F, Slosman D, Junod A, Bounameaux H (1997) D-dimer testing for suspected pulmonary embolism in outpatients. Am J Respir Crit Care Med 156(2):492-496

17. Palareti G, Cosmi B, Legnani C, Tosetto A, Brusi C, lorio A, Pengo V, Ghirarduzzi A, Pattacini C, Testa S (2006) D-dimer testing to determine the duration of anticoagulation therapy. N Engl J Med 355(17):1780-1789

18. Currie PJ, Seward JB, Chan K-L, Fyfe DA, Hagler DJ, Mair DD, Reeder GS, Nishimura RA, Tajik AJ (1985) Continuous wave Doppler determination of right ventricular pressure: a simultaneous

Page 11/12 
Doppler-catheterization study in 127 patients. J Am Coll Cardiol 6(4):750-756

19. De Vries S, Kleijn S, van't Hof A, Snaak H, van Enst G, Kamp O, Breeman A (2010) Impact of high altitude on echocardiographically determined cardiac morphology and function in patients with coronary artery disease and healthy controls. European Journal of Echocardiography 11(5):446-450

\section{Figures}

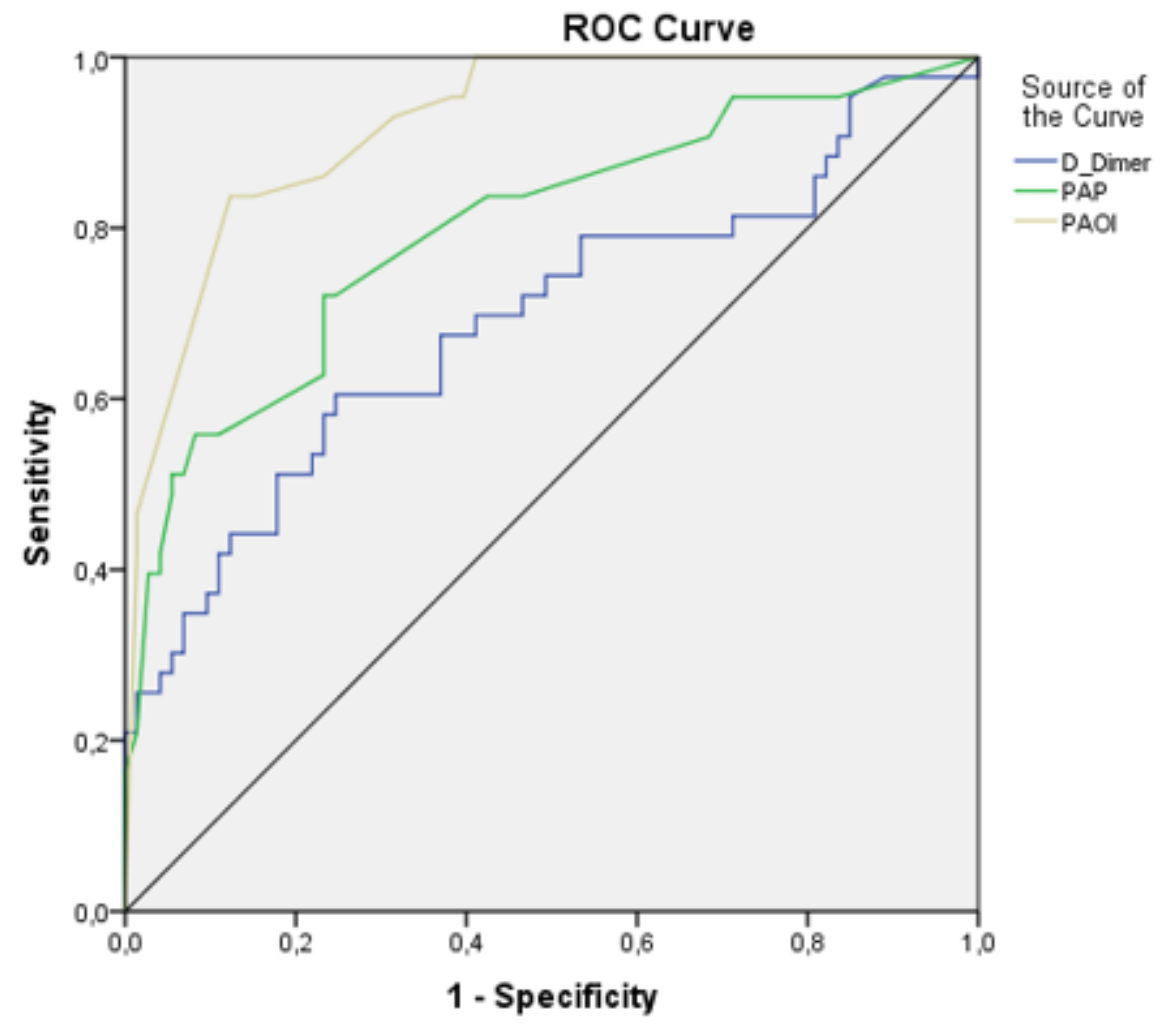

Diagonal segments are produced by ties.

Area Under the Curve

\begin{tabular}{|c|c|c|c|c|c|}
\hline \multirow[t]{2}{*}{ Test Result Variable(s) } & \multirow[t]{2}{*}{ Area } & \multirow[t]{2}{*}{ Std. Error } & \multirow[t]{2}{*}{$\mathrm{p}$} & \multicolumn{2}{|c|}{$\begin{array}{c}\text { Asymptotic } 95 \% \text { Confidence } \\
\text { Interval }\end{array}$} \\
\hline & & & & Lower Bound & Upper Bound \\
\hline D-dimer & .691 & .054 & .001 & .586 & .797 \\
\hline PAP & .799 & .045 & .000 & .711 & .886 \\
\hline PAOI & .921 & .024 & .000 & .873 & .968 \\
\hline
\end{tabular}

PAP: Pulmonary artery pressure, PAOI: Pulmonary artery obstruction index

\section{Figure 1}

Receiver operating characteristic curve analysis for the differentiation of patients with residual or chronic pulmonary thromboembolism and patients with thrombus resolution 